\title{
A note on the equation $x^{y}+y^{z}=z^{x}$
}

\section{Yulin $\mathrm{Lu}^{1}$ and Xiaoxue $\mathrm{Li}^{2 *}$}

\section{*Correspondence:}

Ixx20072012@163.com

${ }^{2}$ School of Mathematics, Northwest University, Xi'an, Shaanxi, P.R. China

Full list of author information is

available at the end of the article

\begin{abstract}
In this paper, we shall use some simple inequalities and a deep result on the existence of primitive divisors of Lucas numbers to prove that the exponential Diophantine equation $x^{y}+y^{z}=z^{x}$ has no positive integer solution $(x, y, z)$ with $2 \mid y$.
\end{abstract}

MSC: $11 \mathrm{D} 61$

Keywords: inequality; exponential Diophantine equation; primitive divisor of Lucas number; existence

\section{Introduction}

Let $\mathbb{Z}, \mathbb{N}$ be the sets of all integers and positive integers, respectively. Recently, Zhang and Yuan [1] were interested in the equation

$$
x^{y}+y^{z}=z^{x}, \quad x, y, z \in \mathbb{N}
$$

Using the Gel'fond-Baker method, they proved that all solutions $(x, y, z)$ of $(1.1)$ satisfy $\max \{x, y, z\}<\exp (\exp (\exp (5)))$. This upper bound is far beyond the computable scope at present. In this paper, we shall use some simple inequalities and a deep result on the existence of primitive divisors of Lucas numbers to prove the following result.

Theorem Equation (1.1) has no solution $(x, y, z)$ with $2 \mid y$.

In addition, it is obvious that $(x, y, z)=(1,1,2)$ is a solution of $(1.1)$. Because one have not found the other solutions, we propose a conjecture as follows:

Conjecture Equation (1.1) has only the solution $(x, y, z)=(1,1,2)$.

Our theorem supports the above mentioned conjecture.

\section{Preliminaries}

Lemma 2.1 Let $f(X)=X / \log X$, where $X$ is a real number. Then $f(X)$ is an increasing function for $X>e$.

Proof Since $f^{\prime}(X)=(\log X-1) /(\log X)^{2}$, we have $f^{\prime}(X)>0$ for $X>e$. Thus, the lemma is proved.

Lemma 2.2 Let $g(X)=\sqrt{X}-2(2+\log (4 X)) / \pi$, where $X$ is a real number. Then we have $g(X)>0$ for $X \geq 16$.

02014 Lu and Li; licensee Springer. This is an Open Access article distributed under the terms of the Creative Commons Attribution License (http://creativecommons.org/licenses/by/2.0), which permits unrestricted use, distribution, and reproduction in any medium, provided the original work is properly cited. 
Proof Since $g^{\prime}(X)=1 / 2 \sqrt{X}-2 / \pi X>0$ for $X \geq 16, \mathrm{~g}(\mathrm{X})$ is an increasing function satisfying $g(X) \geq g(16)>0$ for $X \geq 16$. The lemma is proved.

Lemma $2.3([2,3])$ The equation

$$
X^{2}+2^{m}=Y^{n}, \quad X, Y, m, n \in \mathbb{N}, \operatorname{gcd}(X, Y)=1, n>2
$$

has only the solutions $(X, Y, m, n)=(5,3,1,3)$ and $(7,3,5,4)$.

Lemma 2.4 ([4, Theorem 8.4]) The equation

$$
X^{2}+Y^{m}=2^{n}, \quad X, Y, m, n \in \mathbb{N}, 2 \nmid Y, Y>1, m>1
$$

has only the solution $(X, Y, m, n)=(13,7,3,9)$.

Lemma 2.5 ([4, Theorem 8.4]) The equation

$$
X^{2}-Y^{m}=2^{n}, \quad X, Y, m, n \in \mathbb{N}, 2 \nmid Y, Y>1, m>2, n>1
$$

has only the solution $(X, Y, m, n)=(71,17,3,7)$.

Let $D$ be a positive integer, and let $h(-4 D)$ denote the class number of positive binary quadratic primitive forms of discriminant $-4 D$.

Lemma $2.6 h(-4 D) \leq D$.

Proof Notice that $h(-4)=h(-8)=h(-28)=1, h(-12)=h(-16)=h(-20)=h(-24)=$ $h(-32)=h(-36)=h(-40)=h(-52)=h(-60)=2, h(-44)=3, h(-48)=h(-56)=4$. The lemma holds for $D \leq 15$. By Theorems 11.4.3, 12.10.1, and 12.14.3 of [5], if $D \geq 1$, then

$$
h(-4 D)<\frac{2 \sqrt{D}}{\pi}(2+\log (4 D)) .
$$

Therefore, if $h(-4 D)>D$, then from $(2.4)$ we get

$$
\sqrt{D}<\frac{2}{\pi}(2+\log (4 D))
$$

But, by Lemma 2.2, (2.5) is impossible for $D \geq 16$. Thus, the lemma is proved.

Lemma 2.7 Let $k$ be a positive integer with $\operatorname{gcd}(k, 2 D)=1$. Every solution $(X, Y, Z)$ of the equation

$$
X^{2}+D Y^{2}=k^{Z}, \quad X, Y, Z \in \mathbb{Z}, \operatorname{gcd}(X, Y)=1, Z>0
$$

can be expressed as

$$
\begin{aligned}
& Z=Z_{1} t, \quad t \in \mathbb{N}, \\
& X+Y \sqrt{-D}=\lambda_{1}\left(X_{1}+\lambda_{2} Y_{1} \sqrt{-D}\right)^{t}, \quad \lambda_{1}, \lambda_{2} \in\{ \pm 1\},
\end{aligned}
$$


where $X_{1}, Y_{1}, Z_{1}$ are positive integers satisfying

$$
X_{1}^{2}+D Y_{1}^{2}=k^{Z_{1}}, \quad \operatorname{gcd}\left(X_{1}, Y_{1}\right)=1, Z_{1} \mid h(-4 D)
$$

Proof This lemma is the special case of [6, Theorems 1 and 2] for $D_{1}=1$ and $D_{2}<0$.

Let $\alpha, \beta$ be algebraic integers. If $\alpha+\beta$ and $\alpha \beta$ are nonzero coprime integers and $\alpha / \beta$ is not a root of unity, then $(\alpha, \beta)$ is called a Lucas pair. Further, let $a=\alpha+\beta$ and $c=\alpha \beta$. Then we have

$$
\alpha=\frac{1}{2}(a+\lambda \sqrt{b}), \quad \beta=\frac{1}{2}(a-\lambda \sqrt{b}), \quad \lambda \in\{ \pm 1\},
$$

where $b=a^{2}-4 c$. We call $(a, b)$ the parameters of the Lucas pair $(\alpha, \beta)$. Two Lucas pairs $\left(\alpha_{1}, \beta_{1}\right)$ and $\left(\alpha_{2}, \beta_{2}\right)$ are equivalent if $\alpha_{1} / \alpha_{2}=\beta_{1} / \beta_{2}= \pm 1$. Given a Lucas pair $(\alpha, \beta)$, one defines the corresponding sequence of Lucas numbers by

$$
L_{n}(\alpha, \beta)=\frac{\alpha^{n}-\beta^{n}}{\alpha-\beta}, \quad n=0,1,2, \ldots
$$

For equivalent Lucas pairs $\left(\alpha_{1}, \beta_{1}\right)$ and $\left(\alpha_{2}, \beta_{2}\right)$, we have $L_{n}\left(\alpha_{1}, \beta_{1}\right)= \pm L_{n}\left(\alpha_{2}, \beta_{2}\right)$ for any $n \geq 0$. A prime $p$ is called a primitive divisor of $L_{n}(\alpha, \beta)(n>1)$ if $p \mid L_{n}(\alpha, \beta)$ and $p \nmid b L_{1}(\alpha, \beta) \cdots L_{n-1}(\alpha, \beta)$. A Lucas pair $(\alpha, \beta)$ such that $L_{n}(\alpha, \beta)$ has no primitive divisor will be called an $n$-defective Lucas pair. Further, a positive integer $n$ is called totally nondefective if no Lucas pair is $n$-defective.

Lemma 2.8 ([7]) Let $n$ satisfy $4<n \leq 30$ and $n \neq 6$. Then, up to equivalence, all parameters of $n$-defective Lucas pairs are given as follows:

(i) $n=5,(a, b)=(1,5),(1,-7),(2,-40),(1,-11),(1,-15),(12,-76),(12,-1,364)$.

(ii) $n=7,(a, b)=(1,-7),(1,-19)$.

(iii) $n=8,(a, b)=(2,-24),(1,-7)$.

(iv) $n=10,(a, b)=(2,-8),(5,-3),(5,-47)$.

(v) $n=12,(a, b)=(1,5),(1,-7),(1,-11),(2,-56),(1,-15),(1,-19)$.

(vi) $n \in\{13,18,30\},(a, b)=(1,-7)$.

Lemma 2.9 ([8]) If $n>30$, then $n$ is totally non-defective.

\section{Further lemmas on the solutions of (1.1)}

Throughout this section, we assume that $(x, y, z)$ is a solution of $(1.1)$ with $(x, y, z) \neq(1,1,2)$.

Lemma 3.1 ([1]) $x, y$ and $z$ are coprime.

Lemma $3.2 \min \{x, y, z\} \geq 3$

Proof Since $z^{x}=x^{y}+y^{z}>1$, we have $z>1$. If $x=1$, since $(x, y, z) \neq(1,1,2)$, then $y>1$ and $z=1+y^{z} \geq 1+2^{z} \geq z+3$, a contradiction. Similarly, if $y=1$, then $x>1$ and $x+1=z^{x} \geq 2^{x} \geq$ $x+2$, a contradiction. Therefore, we have $\min \{x, y, z\} \geq 2$.

If $x=2$, then

$$
2^{y}+y^{z}=z^{2}
$$


Further, by Lemma 3.1, $y$ and $z$ are odd integers with $\min \{y, z\} \geq 3$. Hence, we see from (3.1) that (2.3) has the solution $(X, Y, m, n)=(z, y, z, y)$. But, by Lemma 2.5, it is impossible. Similarly, if $y=2$ or $z=2$, then we have

$$
x^{2}+2^{z}=z^{x}, \quad 2 \nmid x z, \min \{x, z\} \geq 3
$$

or

$$
x^{y}+y^{2}=2^{x}, \quad 2 \nmid x y, \min \{x, y\} \geq 3 .
$$

But, by Lemmas 2.3 and 2.4, (3.2) and (3.3) are impossible. Thus, we get $\min \{x, y, z\} \geq 3$. The lemma is proved.

Lemma $3.3 y<x$.

Proof By (1.1), we have $z^{x}>x^{y}$ and $z^{x}>y^{z}$. Hence,

$$
\frac{x}{\log x}>\frac{y}{\log z}
$$

and

$$
\frac{x}{\log y}>\frac{z}{\log z}
$$

In addition, by Lemmas 3.1 and 3.2, $x, y$ and $z$ are distinct.

If $x<y<z$, by Lemma 3.2, then $3 \leq x<y<z$. Hence, by Lemma 2.1, we get

$$
\frac{z}{\log z}>\frac{x}{\log x}>\frac{x}{\log y}
$$

which contradicts (3.5). Similarly, we can remove the case that $x<z<y$.

If $z<x<y$, then $3 \leq z<x<y$ and

$$
\frac{y}{\log z}>\frac{y}{\log y}>\frac{x}{\log x},
$$

which contradicts (3.4). Thus, we get $y<x$. The lemma is proved.

\section{Proof of theorem}

We now assume that $(x, y, z)$ is a solution of $(1.1)$ with $2 \mid y$. Since $(x, y, z) \neq(1,1,2)$, by Lemmas 3.1, 3.2 and 3.3, we have $2 \nmid x z, \operatorname{gcd}(y, z)=1, \min \{x, y, z\} \geq 3$ and $x>y$.

We see from (1.1) that the equation

$$
X^{2}+y Y^{2}=z^{Z}, \quad X, Y, Z \in \mathbb{Z}, \operatorname{gcd}(X, Y)=1, Z>0
$$

has the solution

$$
(X, Y, Z)=\left(x^{y / 2}, y^{(z-1) / 2}, x\right) .
$$


Applying Lemma 2.7 to (4.1) and (4.2), we have

$$
\begin{aligned}
& x=Z_{1} t, \quad t \in \mathbb{N}, \\
& x^{y / 2}+y^{(z-1) / 2} \sqrt{-y}=\lambda_{1}\left(X_{1}+\lambda_{2} Y_{1} \sqrt{-y}\right)^{t}, \quad \lambda_{1}, \lambda_{2} \in\{ \pm 1\},
\end{aligned}
$$

where $X_{1}, Y_{1}, Z_{1}$ are positive integers satisfying

$$
X_{1}^{2}+y Y_{1}^{2}=z^{Z_{1}}, \quad \operatorname{gcd}\left(X_{1}, Y_{1}\right)=1
$$

and

$$
Z_{1} \mid h(-4 y)
$$

Let

$$
\alpha=X_{1}+Y_{1} \sqrt{-y}, \quad \beta=X_{1}-Y_{1} \sqrt{-y} .
$$

We see from (4.5) and (4.7) that $\alpha+\beta=2 X_{1}$ and $\alpha \beta=z^{Z_{1}}$ are coprime nonzero integers, $\alpha / \beta=\left(\left(X_{1}^{2}-y Y_{1}^{2}\right)+2 X_{1} Y_{1} \sqrt{-y}\right) / z^{Z_{1}}$ is not a root of unity. Hence, $(\alpha, \beta)$ is a Lucas pair with parameters $\left(2 X_{1},-4 y Y_{1}^{2}\right)$. Further, Let $L_{n}(\alpha, \beta)(n=0,1,2, \ldots)$ denote the corresponding Lucas numbers. By (4.4) and (4.7), we have

$$
y^{(z-1) / 2}=\left|L_{t}(\alpha, \beta)\right| .
$$

We find from (4.7) and (4.8) that the Lucas number $L_{t}(\alpha, \beta)$ has no primitive divisor. Therefore, by Lemma 2.9, we have $t \leq 30$. Further, since $2 \nmid x$ and $2 \nmid t$ by (4.3), it is easy to remove all cases in Lemma 2.8 and conclude that $t \in\{1,3\}$.

If $t=3$, then from (4.4) we get

$$
y^{(z-1) / 2}=\lambda_{1} \lambda_{2} Y_{1}\left(3 X_{1}^{2}-y Y_{1}^{2}\right)
$$

Let $d=\operatorname{gcd}\left(Y_{1}, 3 X_{1}^{2}-y Y_{1}^{2}\right)$. Since $\operatorname{gcd}\left(X_{1}, Y_{1}\right)=1$, we have $d \mid 3$ and $d \in\{1,3\}$. Further, since $t \mid x$, we get $3 \mid x, 3 \nmid y$ and $d \neq 3$ by (4.9). Therefore, we have $d=1$ and, by (4.9), $\operatorname{gcd}\left(y, 3 X_{1}^{2}-\right.$ $\left.y Y_{1}^{2}\right)=1$ and

$$
Y_{1}=y^{(z-1) / 2}, \quad 3 X_{1}^{2}-y Y_{1}^{2}= \pm 1
$$

It implies that

$$
3 X_{1}^{2} \mp 1=y^{z} .
$$

But, since $2 \mid y$ and $z \geq 3$, we get from (4.11) that $2 \nmid X_{1}$ and $0 \equiv y^{z} \equiv 3 X_{1}^{2} \mp 1 \equiv 3 \mp 1 \not \equiv 0$ (mod 8$)$, a contradiction.

If $t=1$, then from (4.3) and (4.6) that $x=Z_{1}, x \mid h(-4 y)$ and

$$
x \leq h(-4 y)
$$


But recall that $x>y$, by Lemma 2.6, (4.12) is impossible. Thus, (1.1) has no solution $(x, y, z)$ with $2 \mid y$. The theorem is proved.

\section{Competing interests}

The authors declare that they have no competing interests.

\section{Authors' contributions}

$Y L$ obtained the theorems and completed the proof. XL corrected and improved the final version. Both authors read and approved the final manuscript.

\section{Author details}

${ }^{1}$ College of Mathematics and Information Science, Weinan Normal University, Weinan, Shaanxi, P.R. China. ${ }^{2}$ School of Mathematics, Northwest University, Xi'an, Shaanxi, P.R. China.

\section{Acknowledgements}

The authors would like to thank the referee for his very helpful and detailed comments, which have significantly improved the presentation of this paper. This work is supported by the P. S. F. (2013JZ001) and N.S.F. (11371291) of P.R. China.

Received: 11 March 2014 Accepted: 7 April 2014 Published: 07 May 2014

\section{References}

1. Zhang, Z-F, Yuan, P-Z: On the diophantine equation $a x^{y}+b y^{z}+c Z^{x}=0$. Int. J. Number Theory 8(3), 813-821 2012

2. Cohn, JHE: The diophantine equation $x^{2}+2^{k}=y^{n}$. Arch. Math. Basel 59(3), 341-343 (1992)

3. Le, M-H: On Cohn's conjecture concerning the diophantine equation $x^{2}+2^{m}=y^{n}$. Arch. Math. Basel 78(1), 26-35 (2002)

4. Bennett, MA, Skinner, CM: Ternary diophantine equations via Galois representations and modular forms. Can. J. Math 56(1), 23-54 (2004)

5. Hua, L-K: Introduction to Number Theory. Springer, Berlin (1982)

6. Le, M-H: Some exponential diophantine equations I: the equation $D_{1} x^{2}-D_{2} y^{2}=\lambda k^{2}$. J. Number Theory 55(2), $209-221$ (1995)

7. Voutier, PM: Primitive divisors of Lucas and Lehmer sequences. Math. Comput. 64, 869-888 (1995)

8. Bilu, Y, Hanrot, G, Voutier, PM: Existence of primitive divisors of Lucas and Lehmer numbers (with an appendix by M Mignotte). J. Reine Angew. Math. 539, 75-122 (2001)

\section{Submit your manuscript to a SpringerOpen ${ }^{\ominus}$ journal and benefit from:}

- Convenient online submission

- Rigorous peer review

- Immediate publication on acceptance

- Open access: articles freely available online

- High visibility within the field

- Retaining the copyright to your article 